\title{
A Topology Control Algorithm of Wireless Sensor Network Based on Backup Node
}

\author{
$\mathrm{Li} \mathrm{Mi}$ \\ Soft ware College \\ Shenyang Normol University \\ Shenyang, China \\ e-mail:1274139267@qq.com \\ Li Shangpeng \\ Soft ware College \\ Shenyang Normol University \\ Shenyang, China \\ e-mail: limi0400@sina.com
}

\begin{abstract}
Self-organization and limited energy are two features of wireless sensor network. This paper proposes a practice topology control algorithm for wireless sensor network based on adding backup node and hidden link in traditional topology tree. Because of the HRP(Hierarchical Routing Protocol), build links and generate routing information at the same time; therefore this feature can save resource while no wasting resource on maintaining routing information. Furthermore, maintaining backup nodes can provide an inheritable self-repair function, once any node disabled, it can still keep the network connectivity by invoking a specified procedure.
\end{abstract} repair.

Keywords- wireless sensor network ;topology control; self-

\section{INTRODUCTION}

Wireless sensor network is self-organized into a network program .An outstanding network made up by a good topology control algorithm can keep a welldistributed connection density, which can provide fast multi-hop paths for the demand of communication. While local communication fails because of nodes energy consumption, physical corruption or mobile location and so on, topology control algorithm has the ability to discover the failure and active corresponding counter measures to ensure the connectivity of the entire network timely.

\section{RESEARCH BACKGROUND}

The research on topology control of wireless sensor network aims at looking for a connected graph by controlling nodes degree, single-hop communication distance, etc, to ensure full network's connection. follow:

The connected graph must meet the requirements as

1) Any two nodes have one available communication path at least;

2) The node degree (the count of neighbor nodes) should be uniformed;

\author{
Zhao Yongyi \\ Soft ware College \\ Shenyang Normol University \\ Shenyang, China \\ e-mail: 283631215@qq.com
}

3)Single-hop (edge) distance length should be uniformed and as short as possible.

There are already some related studies about this issue, such as SMECN ${ }^{[1]}, \mathrm{DRNG}^{[2]}, \mathrm{CBTC}^{[3]}, \mathrm{STC}^{[4]}, \mathrm{LMST}^{[5]}$, $\mathrm{COMPOW}^{[6]}$ etc. There are also many outstanding research results recently. However, the premise of these studies mostly separated by topology control algorithm and routing algorithm, no matter what the connected graph, the partial connect of the network are only known to these partial related nodes. It means that the demand of communication in wireless sensor networks, that is, seeing from the communication of each node and sink node , a question arises: the communication source node lack of information to find the optimal path. So, if these distributed topology control algorithm needs to be developed, it must be combined with some other routing algorithm, such as $\mathrm{AODV}^{[7]}, \mathrm{DSR}^{[8]}, \mathrm{LAR}^{[9]}, \mathrm{S}-\mathrm{RHR}$, etc. The topology control algorithm runs and generates a fully connected graph after it's established, and this graph becomes the input conditions of the distributed routing algorithm, then obtains the communication path. It turns into two steps work. as the increasing of the network load from creating and maintaining routing information, when network scale becomes very large, an independency of topology control routing algorithm due to the extra burden will be more obvious.

For the current commercial mature protocol stack, for instance, the Zigbee making sink nodes as the center, starshaped and tree-shaped structure as the main topology structure. These topology structures forming connecting relationship by HRP routing manner.But simple treeshaped absences is very obvious, it can be proved simply: when some node disabled, the communication path which uses this node as the relay node will be failed. And the distance of the sink node of closer energy consumption faster, it will fail more quickly, eventually led to the paralysis of network.

To solve this problem the ZigBee solution is adding a mesh network in the tree-shaped topology structure to acquire other path. The implementation method is building 
tree-shaped topology structure, running improvement AODV routing protocol later. But the style of AODV protocol is using the flood way to discover routing, a greater load on the network, and when the middle layer failure nodes need to call other paths generated from AODV, tree-shaped topology efficient path would not be recovered. Most of the research to this issue, studies on the use of new driving route based on ZigBee which takes place of the AODV ${ }^{10]}$, or start from the routing to improve it on the basis of HRP and AODV ${ }^{[11-12]}$.

\section{ALGORITHM DESCRIPTION}

This paper presents an improved topology control algorithm .The core concept of the algorithm is that when the sink node raise from tree-shaped structure, every nodes (except sink nodes) absorb new lower-level nodes, setting the first child node as a backup node, this node exchange the address between two nodes of filiation high-level modes or fraternal, then hidden link is built up. If there's a parent death node appearing, just removing it, activating hidden link. In addition, whenever any node accesses network, it will be allocated a number " $g$ ", the value of the " $\mathrm{g}$ " is equal to the parent node quantitative value plus 1 , the value of sink number always for constant 0 , the value of " $g$ " represent the nodes depth in the tree-shaped structure. As shown in the Fig.1, every node has an active link and a hidden link at least, that is equal to common mesh network.
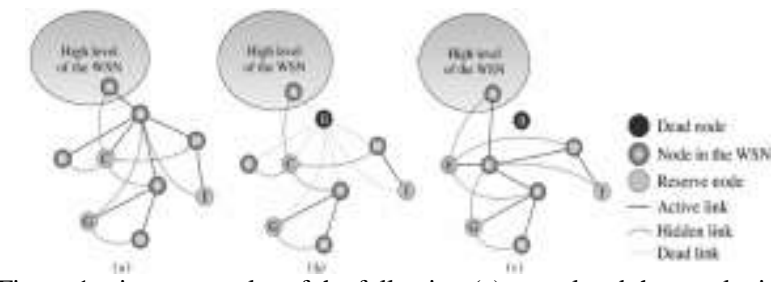

Figure 1. given examples of the following:(a) completed the topological structure ;(b) node B disabled;(c) self-repair results

\section{SELF-REPAIR CHARACTERISTICS}

The node has four states: access network request state, working in the network state, backup nodes working state and handover processing state. Firstly, entering access network request state, after receiving the response, according to parent nodes state entering working state or backup nodes working state, those are normal child nodes or backup nodes. Backup nodes send an requesting message to original nodes at regular intervals, If it doesn't receive any answer at set time, we can concern it as a communication interruption, If interruption occurs multiple times, entering backup nodes handover processing state.

When backup nodes entering handover processing state, firstly, change itself to access network request state. Secondly, finish handover process with hidden link, order high-level nodes delete death nodes, at the same time, update routing. When these steps finished, backup nodes switched into working in the network state, notice death nodes who have other child nodes change the state to access network request state. After notice all nodes, backup nodes change the state to working in the network state.
After child nodes changing to access request state, priority connect with original nodes which has already changed the state. When the distance is too far, it can search for the other access points automatically. If child nodes have lower-level nodes, no longer release and onetime transfer the whole nodes group into the new access point, keep child nodes and lower-level nodes topology state unchanged. In this state, a node group need to update the routing information, after updating, need backup node create a new routing information.

As shown in Fig.2, as the $\mathrm{C}$ is failed, lead to D access backup node $\mathrm{B}$, the local relationship between $\mathrm{D}$ and lower-level is invariant. Taking that into consideration the first hop address of $\mathrm{D}$ and the original routing information on $\mathrm{D}$ is $\mathrm{C}$ in $\mathrm{A}$ and $\mathrm{A}$ 's higher level node. $\mathrm{B}$ is required to issue an upward routing updates to inform A and higherlevel nodes to change the first hop address of D and D's lower node groups into it.

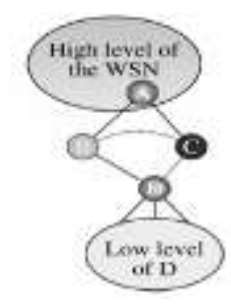

Figure 2.the access of the child node which with lower node cluster at the handover procedures.

In order to ensure the backup node can communicate smoothly when handover, for active connect radius must have constraint conditions. As it is shown in Fig.3, fig.3a shows a common local state diagram, among them, node $\mathrm{B}$ is the original node which has died, C is B's backup node, $\mathrm{A}$ is B's parent node and D is B's child node. If the physics maximum distance is Rmax, the distance of A-B, A-C, B-C, B-D, and C-D are all less than Rmax. As it is shown in picture $3 \mathrm{~b}$, if these nodes distribute in special position, $\mathrm{A}, \mathrm{B}$ and $\mathrm{C}$ in a straight line, when the distance of $\mathrm{AC}$ less than Rmax, the maximum distance of A-B or B-C not more than $\mathrm{Rmax} / 2$.

From the perspective of the whole network, if distributed nodes density regularly and the distribution of node is grid. As it is shown in Fig.3c, every node has 8 neighbor nodes, the farthest neighbors in the grid on the diagonal section and the distance should less than Rmax /2. It means that the grid diagonal length of any node and its 8 neighbor nodes should less than $(\operatorname{Rmax} / \sqrt{2})^{2}$.This grid area is the four nodes in the grid consists of grid vertices and three adjacent grids, four nodes in the edge share with one neighbor grid, So, on the average, each grid gets four points, we can roughly calculate that backup node strategy of node density operation couldn' $t$ less than $(\operatorname{Rmax} / \sqrt{2})^{2}$. 

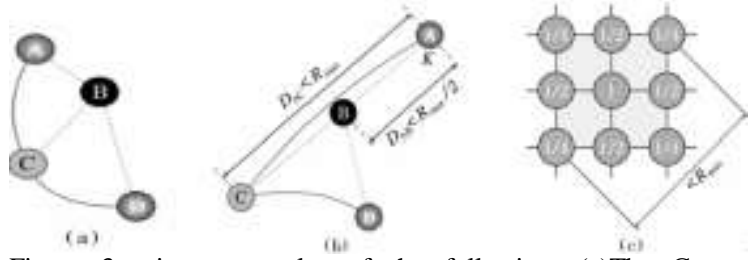

Figure 3. given examples of the following: (a)The Common distribution location ; (b) The special distribution location

If we want to control activation connecting distance, we should judge RSSI of access request packet, when working nodes receive request packet, it can only reply when RSSI value less than the value of the distance Rmax 12 .

\section{SIMULATION EXPERIMENT}

The simulation experiment platform is Matlab and the simulation parameters as the following :set a region which is $500 * 500,300$ nodes were distributed in this region randomly, the aggregation node placed in the center of the region, the limit of communication radius was set as 160 ,the scan radius which used for accept the network is 80. Under the same node deployment status ,comparing basal tree-structured and a DRNG distributed topology control algorithm, generate topological graph and made a statistical about degrees, the relationship of algebra control and degrees, and the distance of single hop communication.

The generated topology structure as shown in Fig.4, Fig.4a is topology structure obtained by DRNG algorithm, Fig.4b is simple tree topology, Fig.4c is the modified tree structure of reverse node mechanism, the imaginary line is implicit connection, $\mathrm{X}$ is ordinary node, the cross is reverse node. Side length network area/m.
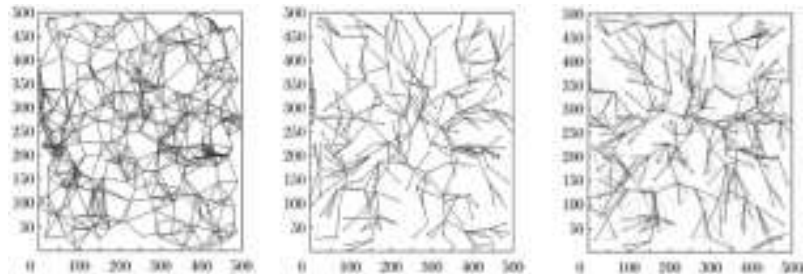

Figure 4 given examples of the following: (a) Topology structure obtained by DRNG algorithm; (b) Simple tree topology;(c) Modified tree structure of backup node mechanism

Extraordinarily, we made a comparison between the degree vs. of simple tree topology and backup strategy tree topology and the average distance vs. of single hop because of the reference to the algebra in the tree structure.

\section{CONCLUSION}

In this paper, the rationality of theory of based on the strategy of backup nodes of a tree topology control algorithm has been proved through the experiment. The given structure of the simulation experiment is only static result after topology has been established, although it can ensure the advantage that relative to the simple tree structure, but because of the effect of nodes density and degree of impact on the sustainability of backup strategy is big, so the dynamic quantitative analysis needs to be perfect.

\section{REFERENCES}

[1]. L.Li,Joseph,Y.H. Minimum-Energy Mobile Wireless Network Revisited[C]//Communication,2001.ICC 2001.IEEEInternational Conference Communications.Helsinki,Finland:IEEE, pp. 278 283,2001 .

[2]. N.Li, Hou.J.C.Localized Topology Control Algorithms for Heterogeneous Wireless Networks[J]. IEEE/ACM Transactions on Networking, 13(6),pp. 1313-1324,2005.

[3]. L.Li, Halpern,J.Y\& Bahl,P., et al. Analysis of a Cone-Based Distributed Topology Control Algorithm for Wireless Multi-hop Networks[C] //Proceedings of the twentieth annual ACM symposium on Principles of distributed computing. New York: ACM, pp. 264-273,2001.

[4]. Sethu,H.,Gerety,T. A Distributed Topology Control Algorithm in the Presence of Multipath Propagation[C]//Fourth Annual International Conference on Mobile and Ubiquitous Systems: Networking\&Service. Philadelphia,PA:IEEE,pp. 1-8,2007.

[5]. Narayanasw,S.A., Kawadia,V. \& Sreenivas ,R.S., et al. Power Control in Ad-Hoc Networks: Theory, Architecture, Algorithm and Implementation of the COMPOW protocol[C]// Proceeding of European Wireless 2002. Next Generation Wireless Networks Technologies, Protocols, Services and Applications. February,pp. 156-162,2002.

[6]. Narayanasw, A.S,Kawadia,V.\&Sreenivas,R.S. Design and Analysis of an MST-Based Topology Control Algorithm[C]//Twenty-Second Annual Joint Conference of the IEEE Computer and Communications. IEEE Societies,pp. 17021712,2003 .

[7]. Perkins,C.E.\&Royer,E.M. Ad-hoc On-Demand Distance Vector Routing[C]//Proceedings. WMCSA 99. Second IEEE Workshop on Mobile Computing Systems and Applications,1999.New Orleans, LA, USA:IEEE,pp. 90-100,1999.

[8]. Johnson,D.,Hu,Y.\&Maltz,D., et al. The Dynamic Source Routing Protocol(DSR) for Mobile Ad Hoc Networks for IPV4[EB/OL].(201203-05)[2012-0318].https://datatracker.ietf.org/doc/rfc4728/? Include text=1.

[9]. Ko,Y.B.,Vaidya,N.H. Location-Aided Routing(LAR) in mobile ad hoc networks[J]. Wireless Networks, 6(4),pp. 307-321,2000.

[10]. Lee,K.K,Kim,S.H.\&Park,H.S.Cluster Lable-based Zigbee Routing Protocol with High Scalability[C]//ICSNS 2007. Second International Conference on Systems and Networks Communications, 2007. IEEE,pp. 12-12,2007.

[11]. Ha,J.Y, Park,H.S\&Chou,S., et al.EHRP:Enhanced Hierarchica Routing Protocol for Zigbee Mesh Networks[J]. IEEE,pp. 10281030,2007(11).

[12]. Ren,P., Sun,M.H.\&Zou,Y.M, et al. ZigBee Routing Selection Strategy Based on Data Service and Energy-Balanced ZigBee Routing [C]//APSCS 06.IEEE Asia-Pacific Conference,pp. 400404,2006. 